\title{
LA CENTRALITÉ LINGUISTIQUE DU FRANÇAIS DANS L'AFFICHAGE PUBLICITAIRE À MÉDÉA EN ALGÉRIE
}

\author{
Djamel HAMIDI \\ Université Mouloud Mammeri de Tizi-Ouzou, Algérie \\ djamelhamidi26@yahoo.fr \\ Article reçu le 28 mars 2016 | révisé depuis le 16 avril 2016 | accepté le 5 juin 2016
}

\begin{abstract}
RÉSUMÉ. Ce travail s'attache à approcher l'affichage publicitaire de point de vue sociolinguistique. Plus précisément, Il s'agit de voir les langues en présence dans l'espace urbain de Médéa. Pour ce faire, sur le plan théorique, nous avons inscrit notre objet d'étude dans le champ de la sociolinguistique urbaine, discipline qui questionne les pratiques sociolangagières dans leur complexité. Sur le plan méthodologique, nous nous appuyons sur un corpus constitué d'affiches publicitaires. Par le biais de ce corpus, nous focalisons notre attention, d'abord, sur la distribution des langues en contexte urbain ; puis, nous nous intéressons à la place de la langue française dans le paysage graphique urbain. Notre intérêt est donc de vérifier le poids des langues affichées dans la ville de Médéa.
\end{abstract}

Mots-clés : appropriation, langue, sociolinguistique.

\begin{abstract}
This work deals with the urban publicity boards from a sociolinguistic point of view. Mainly, it does deal with the languages presented in urban places of Médéa town. For that, theoretically, we have chosen to subscribe our object of study under the urban sociolinguistic field. This later studies sociolinguistic practices with their various complexities. From a methodological side, we have chosen a corpus consists of billboards. Using that corpus, we have focused first on the distribution of the languages on urban spaces, and after that, we have dealt with the place of French in this urban area. So, our objective is to know the value of all the languages in the city of médéa.
\end{abstract}

Keywords: appropriation, language, sociolinguistic.

\section{INTRODUCTION}

"On a défini l'homme comme un animal constructeur de culture (a culture building animal), $c^{\prime}$ est dire que la culture est un attribut universel de l'homme" (Abou, 2002, p. 34).

C'est par cette citation montrant la substance culturelle de l'être humain que nous commençons cet article. L'homme est un être qui essaye de comprendre et de maitriser le monde. Cette compréhension et cette maitrise se font par la voie de ce qu'on nomme «science». Selon ses propres besoins et dans le but d'améliorer son environnement culturel, l'homme, à travers la recherche scientifique, enrichit le monde par la construction d'autres univers.

Définie par Bunge (2001) comme
"[une] connaissance rationnelle,
systématique, exacte, vérifiable et, en
conséquence, faillible"
(p.5), la science peut


être divisée en deux domaines de recherche. L'un est celui du formel et $\mathrm{du}$ rationnel comme les mathématiques et la logique ; l'autre est celui du factuel, qui s'occupe des faits réels. Notre champ de travail, la sociolinguistique, s'inscrit dans le second domaine et propose un espace à large gamme de problématiques.

La sociolinguistique, comme l'a défini Baylon (2008) est "l'étude du langage dans son contexte socioculturel“(p.35). C'est une science qui s'intéresse à des phénomènes variés comme les "usages" de la langue, la planification linguistique, etc. La naissance de cette discipline est due à une sommité dans le domaine de la linguistique qui s'est soulevée contre la forme puriste de la linguistique saussurienne. Par son enquête visant l'étude du rapport entre la classe sociale et les variations en prononciation, Labov (1976) a invité les spécialistes de langues à un nouveau regard sur le langage en leur reprochant le fait que :

ils travaillent dans leur bureau avec un ou deux informateurs, ou bien examinent ce qu'ils savent eux-mêmes de la langue. Qui plus est, ils s'obstinent à rendre compte des faits linguistiques, et refusent toute explication fondée sur des données « extérieures », tirées du comportement social (p.257).

Pourquoi un tel reproche? Crystal (2003) nous répond que "language has no independent existence, living in some sort of mystical space a part from the people who speak it. Language exists only in the brains and mouths and ears and hands and eyes of its users" (p.7).

Baptisée comme une linguistique de terrain, la sociolinguistique s'intéresse à tout ce qui, dans la langue, est relatif au social, ce qui se gère, se règle socialement. Elle se donne pour tâche d'étudier la langue en contexte, les croyances accompagnant son choix, etc.

Le sociolinguiste observe et analyse [...] les variations de la langue, ses divers usages au sein de la communauté linguistique en fonction de variables sociales, sans jamais dissimuler que ces variations, ces usages sont clairement perçus, étiquetés, évalués par les membres de la communauté. (Boyer, 2001, p.45)

Depuis les années 1990, on assiste à l'émergence d'une tendance sociolinguistique française et francophone, initiée par Louis-jean Calvet, qui se veut "urbaine" et cherche la spécificité langagière du contexte urbain. Discipline nouvelle et émergente, la sociolinguistique, urbaine de nom, porte un regard neuf sur la ville en tant qu'espace de signes complexes et dynamiques. Cette focalisation sur la ville est justifiée par le fait qu'elle est un lieu d'hétérogénéité et de pratiques sociolangagières multiples qui "se dresse à l'horizon de notre histoire immédiate comme un inévitable destin" (Calvet, 1994, p.10). Elle est un inévitable destin parce qu'elle attire de plus en plus de mouvements migratoires, source de concentration et symbole de "matérialisation physique des désirs humains: argent, travail, contacts sociaux, loisirs, culture" (Gasquet-Cyrus, 2002, p. 65). En s'interrogeant sur sa distinction d'une sociolinguistique "non-urbaine", Thierry Bulot affirme que la sociolinguistique urbaine est une sociolinguistique en crise et de crise :

En crise parce qu'elle naît de la sociolinguistique et traverse donc son premier questionnement identitaire en s'interrogeant sur ce qui la crée et la constitue, ce qui la relie à sa science fondatrice et ce qui l'en différencie. De crise parce qu'elle reflète, comme la sociolinguistique en général, une société qui l'est tout autant et parce que les valeurs qui la sous-tendent (et guident ceux qui la font progresser) amènent à dépasser l'observation du changement social et à s'interroger quant à un engagement politique (Bulot, 2002, p. 9).

La sociolinguistique urbaine répond à une double crise: la première est celle de comprendre des phénomènes sociolangagiers pour faire avancer la recherche ; la seconde est sociale, celle d'intervenir pour résoudre les problèmes d'exclusion, d'intégration des migrants. C'est pourquoi, la mission du chercheur n'est pas seulement de décrire la ville dans sa pluralité mais aussi il s'agit de discuter entre autres les dynamiques identitaires urbaines, la gestion 
des langues via les signalétiques linguistiques, les dénominations des lieux urbains, etc.

La sociolinguistique urbaine ne poétise pas les faits langagiers; elle les décrit. Mais cette description n'est plus une fin en soi, car l'objectif en est d'analyser les faits observables pour découvrir les mécanismes responsables. Le chercheur en sociolinguistique urbaine ne tâtonne pas dans l'obscurité. Par contre, il travaille sur le terrain, sur des données authentiques.

En sociolinguistique urbaine, l'affichage publicitaire est considéré comme une forme de marquage qui, par ses langues, rend compte du panorama linguistique d'un espace urbain et dévoile certaines traces permettant une lecture multiple de la ville, une lecture explicite d'un rapport de réciprocité liant les pratiques linguistiques aux acteurs sociaux.

Publicité et langues sont les deux notions sur lesquelles nous avons choisi de nous pencher dans le cadre de cet article qui est une contribution à la compréhension d'une pratique langagière nouvellement installée dans la ville de Médéa : l'affichage publicitaire. Si le mot "publicité" s'avère de nature économique, lié au pouvoir d'investissement et de l'argent, entendre le mot "langue(s)" met les Algériens, à chaque occasion, devant de dures polémiques et leur font penser à leur(s) "carte(s) d'identité(s)".

Etant dit, notre problématique s'orchestre sur les deux questions suivantes : (a) Quelles sont les langues en présence dans l'affichage publicitaire en Algérie? ; et (b) Quelle est la place occupée par la langue française dans ce pays?

La réponse à cette problématique impose la reformulation des hypothèses suivantes: (a) L'affichage publicitaire serait plurilingue malgré le monolinguisme de l'Etat algérien; et (b) Le français aurait une place importante dans le paysage publicitaire.

Pour vérifier ces hypothèses, nous avons entrepris une enquête empirique dans l'une des villes algérienne : la ville de Médéa.

\section{MÉTHODE}

Médéa est une ville algérienne qui se situe à $90 \mathrm{Km}$ au sud d'Alger la capitale. Habitée dès le pré- histoire, la ville de Médéa était l'une des villes du royaume de la Numidie. A la fin du premier siècle, elle a été occupée par les romains qui lui ont donnée le nom d'ad Médéa qui signifie au milieu, parce que d'après Haddadou (2012), elle est "placée au milieu de deux villes, Thamaramusa (act. Berrouaghuia) et Sufasar (act. Amourah)"(p.401). Entre Romains et Vandales, la ville a été prisonnière durant longtemps.

Après les conquêtes arabes, Buluggin Ibn Ziri a reconstruit la ville au XXème siècle avant qu'elle ne tombe entre les mains des Almohades et des Mérinides respectivement au 12 ème et 14 ème siècle.

À l'arrivée des Turcs en Algérie, elle est devenue capitale du beylik du Titteri. Prise en 1840 par la colonisation française après une résistance dure de l'Emir Abdelkader, Médéa a été déclarée commune de plein exercice en 17 juin 1854.

Durant la révolution algérienne (19541962), la ville a eu sa part, comme toute la région, de souffrance et de sacrifices. Après l'indépendance, la ville n'était pas dans les préoccupations des dirigeants de l'époque. Sa situation s'est aggravée en pire dans les années quatre-vingt-dix 1990 (la décennie noire) où elle s'est baignée dans le sang. La ville a pris enfin un nouveau souffle vers les années 2000 avec le retour de la stabilité au pays et connait la réalisation de nombreuses infrastructures économiques, éducatives, culturelles,...Cette situation a encouragé les gens à venir s'y installer.

La ville de Médéa connait de plus en plus un accroissement démographique dû à l'exode rural, surtout durant les années 1990, et aussi à la mobilité des gens qui viennent $\mathrm{s}^{\prime} \mathrm{y}$ installer pour travailler. Ces circonstances ont changé d'une façon remarquable le paysage linguistique, et même sociologique, de la ville de Médéa et a fait d'elle une ville plurilingue.

Pour réaliser une enquête au sein de la ville de Médéa, nous avons collecté durant le premier trimestre de l'année 2014 trente deux affiches publicitaires afin de les analyser de 
point de vue sociolinguistique. L'analyse de ces affiches va nous permettre de voir les langues affichées, le poids de chaque langue dans l'environnement publicitaire, et bien sûr voir la place réservée à la langue française, langue considérée par les textes officiels comme langue étrangère.

\section{RÉSULTATS ET DISCUSSION}

Avant de discuter les résultats de notre enquête, nous voulons faire un petit rappel historique quant à la situation linguistique de l'Algérie. A l'aube de la colonisation française en 1830, le français a été imposé aux Algériens pour désarabiser les Algériens et les déraciner de leur algérianité, et donc, accomplir le processus d'assimilation et /ou d'acculturation et démolir les substrats de l'identité algérienne symbolisée entre autres par la langue arabe, l'islam, etc. Pour cela, des opérations de destructions des écoles coraniques, berceaux de la langue arabe et de l'islam, et des mosquées ont été largement menés par les autorités françaises de l'époque. Le français est, comme l'explique Hagège (2008), la facette culturelle de la colonisation, "le français était conçu comme une arme importante pour soutenir une politique d'expansion puisqu'il était offert aux colonisés comme la face culturelle, c'està-dire, à longue échéance, la plus profitable, d'une cynique action commerciale" (p.24).

$\mathrm{Au}$ lendemain de l'indépendance, l'arabe standard a été désigné la seule langue officielle et nationale. L'officialisation consiste à inscrire le pays dans la nation arabe sous l'influence de l'idéologie panarabe et nationaliste défendue par les baathistes du Moyen Orient dont le chef historique de l'Égypte Djamel Abdenacer.

Le système politique, par la voix de l'arabisation, revendication nationaliste ancienne, selon Taleb-Ibrahimi (1997) veut intégrer le pays "à la sphère civilisationnelle et culturelle qui est celle de la Nation Arabe (Oumma) et de la communauté musulmane en prenant à son compte, en continuité des luttes menées par le Mouvement National" (p.185).

Ces propos de Taleb-Ibrahimi, éclairent l'influence qu'ont deux courants idéologiques sur la politique linguistique : le nationalisme arabe et l'islamisme politique. Le nationalisme arabe, promu par Djamal Abdenacer (1918-1970), affiche pour objectif de réunir les pays arabes sous un seul État arabe pour contrer le colonialisme et sortir du sous-développement. Dans ce projet, la langue arabe participe fortement $\mathrm{vu}$ la sacralité que ses locuteurs lui attribuent et son caractère jugé inimitable. L'islamisme politique s'est proclamé comme solution alternative après la défaite du panarabisme notamment après les guerres de 1967 et 1973 contres Israël.

L'arabisation, comme choix idéologique et linguistique, repose sur quatre arguments (Laroussi, 2003) qui ne manquent pas d'importance.

- Un argument d'ordre religieux qui voit en langue arabe son caractère « divin» un élément unificateur de la «Umma-a »

- Un argument d'ordre historique, d'où la volonté de ressusciter l'Age d'or de la civilisation arabo-musulmane.

- Un argument d'ordre culturel se basant sur le patrimoine culturel arabe, produit de la fusion de plusieurs civilisations.

- Un argument d'ordre idéologique considérant l'arabe comme la face culturelle de l'indépendance, et prise tel" le ciment qui solidifie les liens entre les différents pays de la Nation arabe, pays qui peuvent différer par les coutumes, des aspects culturels, le régime politique...mais sont unifiés par la langue (p.144).

Féconde d'enjeux politiques, sociaux, symboliques, la politique d'arabisation, selon les décideurs de l'époque, est vue comme refuge pour exprimer l'identité algérienne et son appartenance arabo-musulmane ; bref, comme l'indique Haddadou (2003), "le choix de l'arabe classique est justifié par la nécessité d'opposer au colonialisme une langue au passé prestigieux mais aussi une langue unitaire, qui ne connaît pas, parce qu'elle est figée, le foisonnement dialectal de l'arabe parlé ou du berbère" (p.133).

La politique d'arabisation a été largement critiquée à cause de son essence homogénéisant, écartant la diversité culturelle et linguistique, surtout de la part 
des militants berbérophones qui ont pu en avril 2001, suite à des revendications enracinées dans la crise berbère de 1949, arracher le statut de langue nationale pour la langue berbère, langue des populations autochtones du Maghreb.

Néanmoins, le français a gardé un statut privilégié non toujours déclaré, surtout dans l'enseignement supérieur et dans l'administration malgré les différentes vagues d'arabisation. Cela se passe au même moment que les grands efforts faits par la France, au sein de l'Organisation Internationale de la Francophonie, pour promouvoir le français et sa culture. A cet égard, Hagège (2008) espère que "le projet francophone devrait acquérir une dimension qui aille bien au-delà de la langue, puisqu'il intégrerait une construction politique et une entreprise de développement durable" (p.152).

Revenons aux résultats de notre recherche. Dans le corpus étudié, il y a deux marchés publicitaires: l'un est national, composé de deux langues génétiquement parentales qui sont l'arabe standard et l'arabe dialectal ; l'autre est international, formé de deux langues politiquement étrangères (le français et l'anglais), ce qui confirme la diversité linguistique de l'environnement graphique algérien et sa position en décalage avec les directives étatiques le concernent: $C^{\prime}$ est la rupture entre la politique linguistique in vivo et la politique linguistique in vitro.
Le terme politique linguistique, selon Calvet (1999), désigne "l'ensemble des choix conscients effectués dans un domaine des rapports entre langue et vie sociale et plus particulièrement entre langue et vie nationale..." (p.154-155). Ces choix conscients, à un niveau étatique, s'effectuent bien évidemment par une compétence ayant le pouvoir et les moyens de prendre des décisions en matière de langues et de leurs statuts et qui dépend d'intérêts d'un ordre soi-disant identitaire, autrement-dit pour assurer l'unicité nationale et identitaire. Ce type de politique est désigné par Calvet sous le nom de "politique linguistique in vitro".

Face à ce premier type de politique linguistique traduisant "l'intervention directe et volontaire du pouvoir politique dans le domaine linguistique" (Calvet, 1999, p.153), il y a un second qui va à l'opposée du premier et qui est celui de la communauté sociale et ses pratiques socio-langagières quotidiennes: "la politique linguistique in vivo".

La politique linguistique in vivo relève des pratiques effectives des locuteurs qui n'obéissent plus aux lois et décrets législatifs. "Spontanée" et informelle, c'est à la seule loi des exigences de diverses situations de communication qu'elle $\mathrm{s}^{\prime}$ incline.

Concernant les affiches recensées, nous avons relevé treize les résultats suivants :

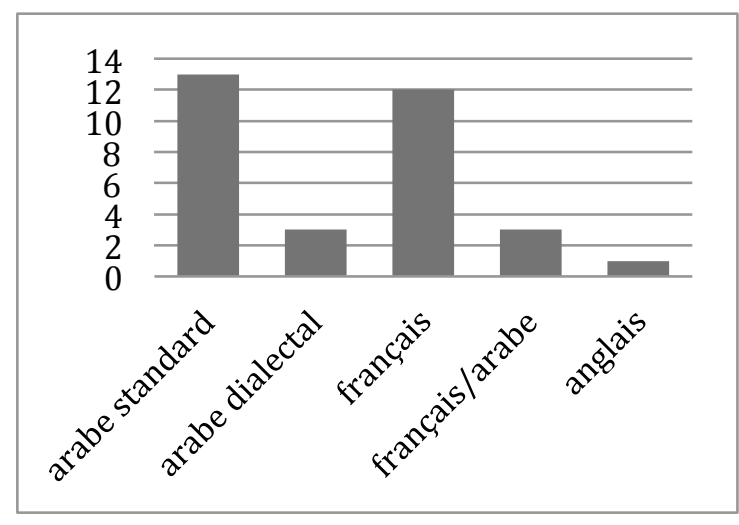

Image 1. Répartition des langues dans la publicité

Treize (13) affiches sont en arabe standard dont la majorité a affaire à valoriser l'appartenance nationale et religieuse comme l'affiche conçue pour inciter à protéger la 
nature ou celle qui vise à glorifier l'Algérie et son équipe nationale. Le français occupe la deuxième place avec douze (12) affiches, et qui véhiculent des produits de luxe, de prestige liés au développement technologique. En troisième place, nous avons trouvé l'arabe dialectal qui promeut des produits de large consommation ou de consommation quotidienne avec trois affiches. Avec le même nombre d'affiche, le bilinguisme français/arabe prouve sa présence où le texte en arabe traduit celui en français et vice-versa. En dernière place, l'anglais bénéficie d'une seule affiche.

Ces résultats nous incitent à dire que les langues dans la ville de Médéa n'ont pas le même "poids". Le terme "poids", énoncé par les frères Calvet, ne renvoie pas seulement à une dimension quantitative d'une langue reposée sur le nombre de ces locuteurs, mais aussi sur sa face qualitative. En commentant la métaphore des frères Calvet, Blanchet (2009) écrit :

D'une part, on inscrit bien les langues parmi les phénomènes humains et sociaux au lieu de les considérer comme des "objets" indépendants des actions humaines [...]. D'autre part, et ceci découle de cela, on investit alors les langues, l'ensemble des pratiques linguistiques, de "valeurs symboliques " culturelles, identitaires, sentimentales, phénomènes massivement répandus chez les humains et qu'il faut savoir prendre en compte. (p.76).

Et note que cette dernière rend compte $\mathrm{du}$ rapport que chaque langue entretient avec d'autres langues, de sa vitalité et son efficacité. Celles-ci dépendent d'abord de sa capacité à produire une culture littéraire féconde, et ensuite de son utilité sociale qui se manifeste dans les pratiques quotidiennes (administration, système scolaire, média, ...).

Cette métaphore de "poids de langue", beaucoup discutée par les sociolinguistes et vue comme métaphore biologique loin du métalangage scientifique spécialisé de la sociolinguistique, a cependant le mérite de poser la question des représentations sociolinguistiques non seulement comme un ensemble de "connaissances qui déterminent la nature des relations qu'entretiennent les locuteurs avec leur(s) langue(s), mais également avec celle des autres communautés" (Gasquet-Cyrys et Petitjean, 2009, p.19).

Les affiches en cette langue sont parsemées dans des lieux très fréquentés comme tout au long de la route nationale $\mathrm{n}^{\circ} 18$, ou dans des lieux à grande importance tels que le pôle universitaire, les centres commerciaux, des institutions administratives. Étant dit, le français est territorialisé dans des lieux de référence, généralement dans le centre-ville.

Et donc, elle est la centralité linguistique, concept qui, selon Bulot, (cité dans Bulot, 2005), "signifie l'attitude qui consiste à placer en un lieu, pour le cas urbain, la forme de prestige sans pour autant que soit attestée la dite forme" (p.231). Et c'est autour du français que gravitent spatialement et, même symboliquement, les autres langues qui dessinent, elles aussi, leurs territoires. Ces langues s'approprient l'espace.

La centralité linguistique du français montre sa vitalité et son omniprésence en Algérie. Nous rejoignons ici Temmim (2007) quand elle écrit que le français est vu comme "signe de promotion sociale et d'ouverture à la modernité. Le français est considéré comme une source d'enrichissement, d'épanouissement et véhicule des valeurs où beauté et prestige prédominent" (p.30).

\section{CONCLUSION}

Pour conclure, nous pouvons dire que l'affichage publicitaire en Algérie se trouve en décalage par rapport la politique linguistique qui fait de la société algérienne une société monolingue. Derrière l'affichage publicitaire, se profile un plurilinguisme motivé par l'envie de promouvoir le produit au plus grand nombre de public possible et des enjeux culturels faisant des langues affichées porteuses à la fois de l'identité culturelle du produit et de l'identité collective des acteurs sociaux.

De plus, faire une publicité dans la langue de l'autre, c'est emprunter les mots de l'autre, avec leurs charges culturelles et, donc, trouver une voix autre que celle de la 
langue maternelle ou officielle. Cette voix, adoptée ou imposée volontairement.

\section{REMERCIEMENTS}

Je tiens à remercier $M$. Ahmed BOUALILI, docteur en sciences du langage, qui m'a aidé, par ses conseils et remarques, dans la réalisation de cette modeste recherche.

\section{RÉFÉRENCES}

Abou, S. (2002). L'identité culturelle, suivi de Cultures et droits de l'Homme. Beyrouth: Éditions Perrin et Les Presses de l'Université Saint-Joseph.

Baylon, C. (2008). Sociolinguistique: société, langue et discours (2e éd.). Paris: Armand Colin.

Blanchet, P. (2009).Gravité et relativité du pesage des langues: avantage, inconvénients et limites d'une métaphore. Dans Gasquet-Cyrus, Médéric et Petitjean, C. (sous dir.). (2009). Le poids des langues (p. 75-88). Paris : L'Harmattan.

Bulot, T. (2002). La sociolinguistique urbaine: une sociolinguistique de crise? Premières considérations. Revue Marges Linguistiques, 3, 8-10.

Bulot, T. (2005). Discours épilinguistique et discours topologique: une approche des rapports entre signalétique et confinement linguistique en sociolinguistique urbaine. Revue de l'Université de Moncton, 36 (2), 219-253. Repéré http://id.erudit.org/iderudit/011994ar

Bunge, M. (2001). La science, sa méthode et sa philosophie. Paris: Vigdor.

Calvet, L-J. (1994).Les voix de la ville: Introduction à la sociolinguistique urbaine. Paris : Payot.
Calvet, L-J. (1999). La guerre des langues. Paris : Hachettes.

Crystal, D. (2003). English as a global language. Cambridge: Cambridge University Press.

Gasquet-Cyrus, M. (2002). Sociolinguistique urbaine ou urbanisation de la sociolinguistique. Revue Marges Linguistiques, 3, 54-71.

Gasquet-Cyrys, M. et Petitjean, C. (2009). La métaphore du poids des langues et ses enjeux. Dans Gasquet-Cyrys, M. et Petitjean (dir.), C. (2009). Le poids des langues (p.9-34). Paris : L'Harmattan.

Haddadou, M-A. (2003). L'Etat algérien face à la revendication berbère: De la répression aux concessions. Revue Glottopol, 1(1), 131-138. Repéré à http://www.univrouen.fr/dyalang/glottopol.

Haddadou, M-A. (2012). Dictionnaire toponymique et historique de l'Algérie. Tizi-Ouzou : Éditions Achab.

Hagège, C. (2008). Combat pour le français. Paris : Odile Jacob.

Labov, W. (1976). Sociolinguistique. Paris: Minuit.

Laroussi, F. (2003). Glottopolitique, idéologies linguistiques et État-nation au Maghreb. Revue Glottopol, 1(1), 139150. Repéré à http://www.univrouen.fr/dyalang/glot topol.

Taleb-Ibrahimi, K. (1997). Les Algériens et leur (s) langue (s). Alger : El Hikma.

Temmim, D. (2007). Nomination et représentation des langues en Algérie. Dans Cheriguen, F. (dir.), Les enjeux de la nomination des langues dans l'Algérie contemporaine (p.19-35). Paris: L'Harmattan. 